\title{
Cervical myeloradiculopathy as an initial presentation of pseudogout
}

Jen-Hsien Liao, MD, Kuo-Chang Huang, MD, Cheng-Ta Hsieh, MD, PhD, Jui-Ming Sun, MD.

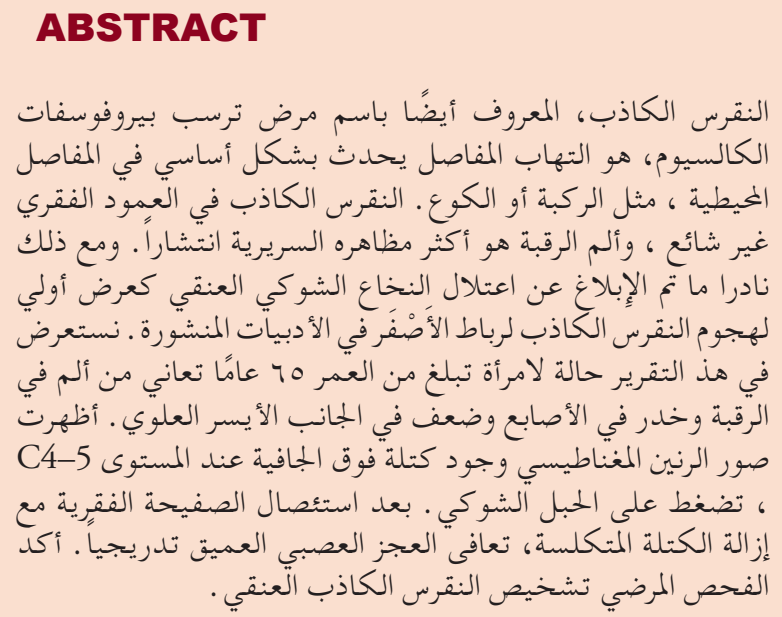

Pseudogout, also known as calcium pyrophosphate deposition disease, is an inflammatory arthropathy that primarily occurs in the peripheral joints, such as the knee or elbow. Spinal pseudogout is uncommon, and neck pain is its most common clinical manifestation. However, cervical myeloradiculopathy as an initial presentation of pseudogout attack of the ligamentum flavum has rarely been reported in the literature. We report a case of a 65-year-old woman who presented with neck pain, bilateral finger numbness, and leftsided upper extremity weakness. Magnetic resonance images showed an epidural mass at the C4-5 level, compressing the spinal cord. Following laminectomy with removal of the calcified mass, the profound neurologic deficits gradually recovered. A pathological examination confirmed the diagnosis of cervical pseudogout.

Neurosciences 2021; Vol. 26 (1): $93-96$ doi: 10.17712/nsj.2021.1.20200122

From the Department of Anesthesiology (Liao), Division of Neurosurgery (Hsieh), Department of Surgery Sijhih Cathay General Hospital, from the Department of Medicine (Hsieh), School of Medicine, Fu Jen Catholic University, New Taipei, from the Department of Neurological Surgery (Hsieh Sun), Tri-Service General Hospital, National Defense Medical Center, New Taipei, from the Section of Neurosurgery (Huang, Sun), Department of Surgery, Ditmanson Medical Foundation, Chia-Yi Christian Hospital, Chia-Yi, and from the Department of Biotechnology (Sun), Asia University, Taichung, Taiwan
Received 21th July 2021. Accepted 18th August 2020.

Address correspondence and reprint request to: Dr. Jui-Ming Sun, Section of Neurosurgery, Department of Surgery, Ditmanson Medical Foundation, Chia-Yi Christian Hospital, Chiayi City, Taiwan. E-mail:07178@cych.org.tw

ORCID: https://orcid.org/0000-0003-2698-5103

$\mathrm{P}$ seudogout, referred to as calcium pyrophosphate deposition (CPPD) disease, is a form of gout-like arthritis that is resistant to digestion by uricase and has no presence of gout. ${ }^{1}$ Cartilage is the major tissue affected by pseudogout, and the knee is the most commonly involved joint, followed by the wrist. ${ }^{1,2}$ However, cord compression caused by a calcified ligamentum flavum from pseudogout attack in the cervical spine has rarely been reported in the literature. ${ }^{2-6}$ We report a case of cervical myeloradiculopathy caused by a calcified ligamentum flavum as an initial presentation of pseudogout.

Case Report. Patient information. This case report was approved by the Institutional Review Board of Chia-Yi Christian Hospital in Taiwan (IRB Number: 2020046). The timeline for this case report was summarized in Table 1. A 65-year-old woman presented with neck pain, bilateral finger numbness, and left-sided upper extremity weakness for 6 months before admission. Her medical history only included hypertension, and her past surgical history only included neurolysis for bilateral carpal tunnel syndrome.

Clinical findings. A neurological examination revealed bilateral hypoesthesia of all digits of the hands and arms, in addition to grade-4 (of 5) muscle power in the left upper extremity. The biceps, triceps, and

Disclosure. The authors declare no conflicting interests, support or funding from any drug company. 


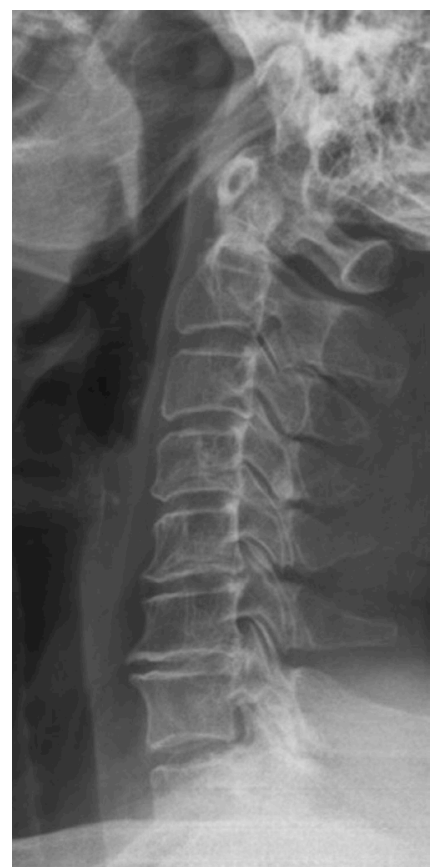

Figure 1 - Lateral view of a cervical radiograph showing marginal spur formation at the C5-6 and C6-7 levels.

brachioradialis deep tendon reflexes were all slightly greater than normal. Bilateral Hoffmann signs were present. Laboratory examination showed white blood cell count of $5900 / \mu \mathrm{L}$ and uric acid level of $4.3 \mathrm{mg} / \mu \mathrm{L}$ (normal range: 2.5 to $6.0 \mathrm{mg} / \mathrm{dl}$ ).

Diagnostic assessment. Radiographs of the cervical spine revealed spondylotic spur formations from the C5 to C7 levels (Figure 1). Magnetic resonance imaging (MRI) of the cervical spine revealed multiple herniated discs at $\mathrm{C} 3$ to $\mathrm{C} 7$ and an epidural mass at the C4-5 level, which was severely compressing the spinal cord. This epidural mass appeared as hypointensity on T1and T2-weighted images (Figure 2), mainly located in the posterior aspect of the spinal canal (Figure 3).

Therapeutic intervention. Upon receiving a diagnosis of cervical myeloradiculopathy associated with an epidural mass, the patient underwent posterior laminectomy of the $\mathrm{C} 4$ level to remove the epidural mass and decompress the spinal cord. At operation, a white and calcified substance intertwined with the ligamentum flavum at the $\mathrm{C} 4$ level was totally removed (Figure 4). This mass did not involve the dura mater or facet joint. Pathological examination with a polarized light microscope revealed deposits of short, rhomboid crystals (calcium pyrophosphate) in fibrous tissue in addition to surrounding fibroblasts and calcified spots, which were compatible with the diagnosis of
(A)
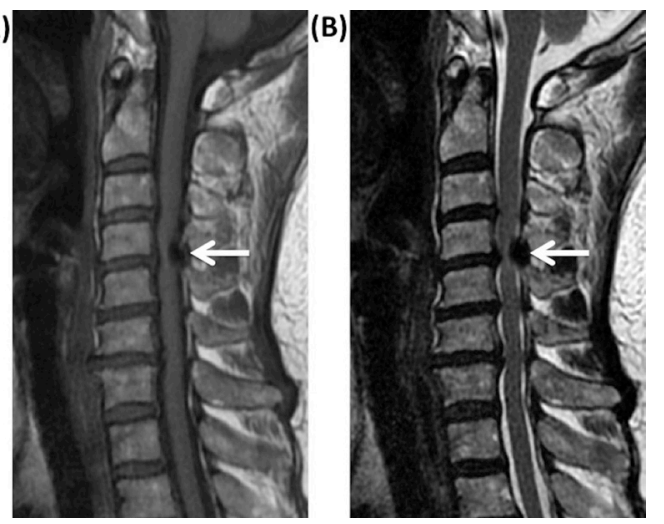

Figure 2 - Sagittal views of magnetic resonance images showing the epidural mass (white arrow), located at the C4-5 level, appearing as hypointensity on the A) T1- and B) T2-weighted images.

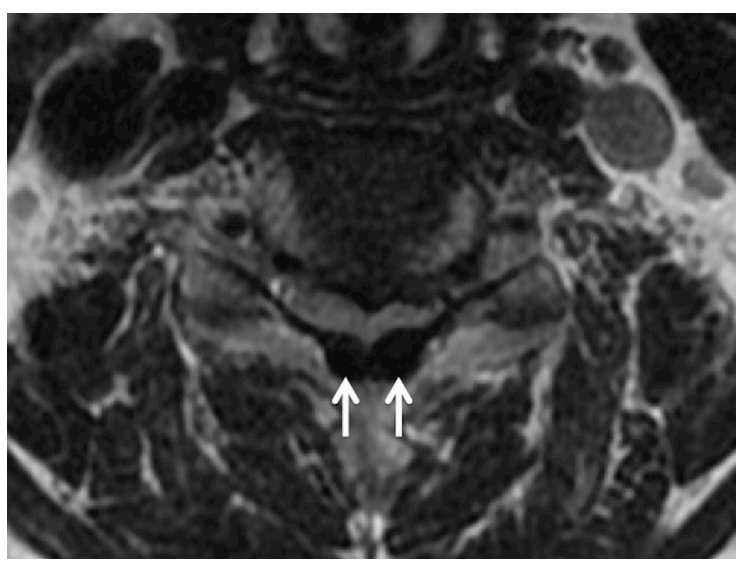

Figure 3 - Axial view of a T2-weighted magnetic resonance image showing the epidural mass (white arrows), which was mainly located in the posterior aspect of the spinal canal around the ligamentum flavum.

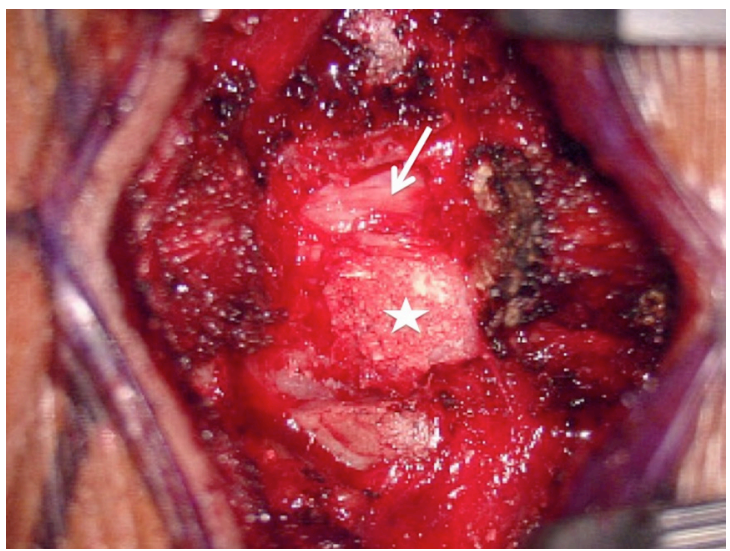

Figure 4 - Intraoperative view showing that the spinal cord (white arrow) was severely compressed by the white and calcified substance that was intertwined with the ligamentum flavum (white asterisk) following laminectomy. 


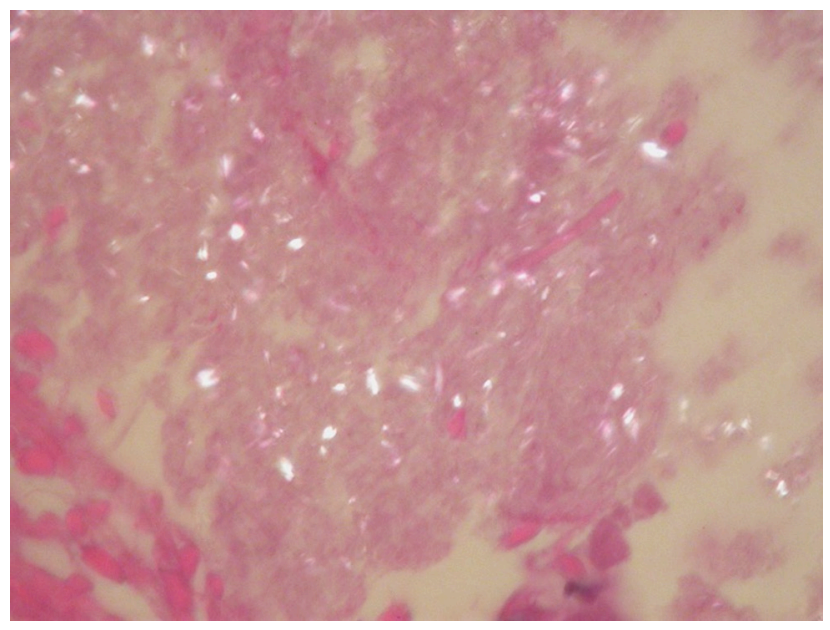

Figure 5 - Polarized light-microscopy view showing deposits of short and rhomboid crystals (calcium pyrophosphate), which were compatible with the diagnosis of pseudogout.

pseudogout (Figure 5).

Follow-up and outcomes. Postoperatively, the motor strength of the left upper extremity fully recovered. Only bilateral fingertip numbness was observed during the patient's visits to a regular outpatient department.

Discussion. The CPPD disease, also known as pseudogout, is an inflammatory arthropathy caused by the deposition of calcium pyrophosphate dehydrate crystals, which are associated with dysfunctions in several transporter and phosphatases. ${ }^{1}$ The prevalence of CPPD disease increases with age, and it is rare in patients aged younger than 50 years. ${ }^{7,8}$ Risk factors for CPPD disease include hyperparathyroidism, osteoarthritis, and loop diuretic use. ${ }^{9}$ CPPD disease tends to be considered a disorder of the peripheral joints, and phosphate crystallization can occur in the articular cartilage, tendons, and ligaments. ${ }^{10}$ However, spinal pseudogout is uncommon, and it has been found to involve the intervertebral disc, synovial joint, articular cartilage, or ligaments. ${ }^{7}$ Most instances of pseudogout occur in the transverse ligament of the atlas, leading to the development of crowned dens syndrome. ${ }^{11} \mathrm{At}$ present, spinal cord compression caused by pseudogout of the ligamentum flavum in the cervical spine, as in our case, has rarely been reported in the literature. ${ }^{2-6}$

The CPPD disease may affect only the spine, but its involvement is commonly asymptomatic. ${ }^{10}$ The clinical manifestation of pseudogout in the cervical spine depends on the severity of affected neural structures. In a prevalent case-control study of the cervical spine in CPPD disease, Finckh et $\mathrm{al}^{8}$ reported neck pain and discomfort to be the most common symptoms in patients with spinal pseudogout. Furthermore, in a retrospective study of 14 patients with cervical pseudogout, Sekijima et $\mathrm{a}^{12}$ noted acute posterior neck pain and fever to be the chief symptoms. In one study, the severity of pain was significantly and positively correlated with the extent of cervical calcified deposits. ${ }^{8}$ Radiculopathy, myelopathy, or myeloradiculopathy may develop when those calcified ligaments formed by pseudogout collectively result in a large compression of the nerve roots or spinal cord. ${ }^{7}$ However, cervical myeloradiculopathy caused by a calcified ligamentum flavum from pseudogout attack has rarely been reported. ${ }^{2-6}$ In a literature review of 26 published cases of compressive cervical myelopathy from pseudogout involving the ligamentum flavum, Fye et $\mathrm{al}^{2}$ reported that $80 \%$ of cases of the disease were in women (mean age: 71.4 years; mean duration between onset of symptoms of cervical myelopathy and diagnosis: 7.7 months). Our present case also involved an old woman (age: 65 years) who had symptoms of cervical myeloradiculopathy for 6 months.

Chondrocalcinosis, the deposition of CPPD crystals into the fibrous or hyaline cartilage, is a common finding in radiographic studies, including those employing X-ray or computed tomography (CT) scans. ${ }^{10}$ Pseudogout can occur with no radiological indication of chondrocalcinosis. ${ }^{1}$ Among patients with CPPD disease, $70 \%$ exhibit calcified deposits in their upper cervical spine, especially in the transverse ligament of the atlas. ${ }^{8}$ For the diagnosis of calcified ligamentum flavum with cord compression, the use of CT and MRI is crucial for confirming which areas are calcified and which neural structures are affected. ${ }^{7,13}$ The differential diagnosis of epidural calcification include those for pseudogout, gout, ossification of the ligamentum flavum, amyloidosis, and hyperparathyroidism associated with chronic renal failure or tuberculosis. ${ }^{14}$ However, the diagnosis of calcium pyrophosphate crystals only can be confirmed through findings from a light microscope, compensated polarized light microscope, or phase contrast microscope-specifically through indications of a characteristic parallepipedic form and intracellular predominance with absent or weak positive birefringence..$^{1,10}$

Surgical decompression is the gold standard treatment for patients with cord compression from a calcified cervical ligamentum flavum. ${ }^{2}$ Laminectomy alone with the removal of the calcified mass has been the common procedure in published cases, and most patients have had a positive outcome, as with our case. ${ }^{2-6}$ If these crystals involve the cervical facet joint, posterior fusion is required to prevent spinal instability. 
The calcified ligamentum flavum may tend to adhere to the dura mater, and postoperative cerebrospinal fluid leaks are likely to be troublesome during the separation of the ossified lesion from the dura. In our case, because the calcified ligamentum flavum did not adhere to the dura, the ossified lesion could be removed with the en bloc method.

In conclusion, although CPPD disease is a common aging-related disorder, cervical myeloradiculopathy may be an initial presentation of a calcified ligamentum flavum from pseudogout attack. Early surgical decompression and microscopic examinations can mitigate the profound neurological deficits induced by the disease and provide an accurate diagnosis.

\section{References}

1. Rosenthal AK, Ryan LM. Calcium Pyrophosphate Deposition Disease. N Engl J Med 2016; 374: 2575-2584.

2. Fye KH, Weinstein PR, Donald F. Compressive cervical myelopathy due to calcium pyrophosphate dihydrate deposition disease: report of a case and review of the literature. Arch Intern Med 1999; 159: 189-193.

3. Roet M, Spoor JK, de Waal M, Kros MJ, Harhangi SB, Dammers R. Extensive calcification of the ligamentum flavum causing cervical myelopathy in a Caucasian woman. Springerplus 2016; 5: 1927.

4. Kawano N, Yoshida S, Ohwada T, Yada K, Sasaki K, Matsuno T. Cervical radiculomyelopathy caused by deposition of calcium pyrophosphate dihydrate crystals in the ligamenta flava. Case report. J Neurosurg 1980; 52: 279-283.

5. Imai S, Hukuda S. Cervical radiculomyelopathy due to deposition of calcium pyrophosphate dihydrate crystals in the ligamentum flavum: historical and histological evaluation of attendant inflammation. J Spinal Disord 1994; 7: 513-517.
6. Chen CF, Chang MC, Wang ST, Liu CL, Chen W. Calcium pyrophosphate dihydrate crystal deposition disease in cervical radiculomyelopathy. J Chin Med Assoc 2003; 66: 256-259.

7. Turaga S, Thomas M, Savy L, Schreiber BE. Pseudogout or pseudolymphoma? Calcium pyrophosphate deposition disease of the cervical spine: a rare presentation and literature review. BMJ Case Rep 2019; 12.

8. Finckh A, Van Linthoudt D, Duvoisin B, Bovay P, Gerster JC. The cervical spine in calcium pyrophosphate dihydrate deposition disease. A prevalent case-control study. J Rheumatol 2004;31:545-549.

9. Rho YH, Zhu Y, Zhang Y, Reginato AM, Choi HK. Risk factors for pseudogout in the general population. Rheumatology (Oxford) 2012; 51: 2070-2074.

10. Rosales-Alexander JL, Balsalobre Aznar J, Magro-Checa C. Calcium pyrophosphate crystal deposition disease: diagnosis and treatment. Open Access Rheumatol 2014; 6: 39-47.

11. Heck A, Nolan N, Rojas-Moreno C. Crowned Dens Syndrome: Calcium Pyrophosphate Deposition Disease Masquerading as Osteomyelitis. J Rheumatol 2018; 45: 1422-1423.

12. Sekijima Y, Yoshida T, Ikeda S. CPPD crystal deposition disease of the cervical spine: a common cause of acute neck pain encountered in the neurology department. J Neurol Sci 2010; 296: 79-82.

13. Kobayashi T, Miyakoshi N, Abe T, Abe E, Kikuchi K, Noguchi $\mathrm{H}$, et al. Acute neck pain caused by pseudogout attack of calcified cervical yellow ligament: a case report. J Med Case Rep 2016; 10: 133.

14. Wada N, Yamashita K, Hiwatashi A, Togao O, Kamei R, Momosaka D, et al. Calcium pyrophosphate dihydrate crystal deposition disease of the spinal dura mater: a case report. BJR Case Rep 2018; 4: 20170049. 